\title{
Fast and Secure Packet Processing Environment for Per-Packet QoS Customization
}

\author{
Takashi Egawa, Koji Hino, and Yohei Hasegawa \\ NEC Corporation \\ 4-1-1 Miyazaki, Miyamae-ku, Kawasaki, 216-8555 Japan \\ t-egawa@ct.jp.nec.com
}

\begin{abstract}
To allow end users define network behavior and to make communication network evolution service-driven, we propose fast and secure capsule processing environment, dedicated CPU architecture named StreamCode and related mechanisms. In our proposed architecture each packet contains a program for the packet written in StreamCode binary instructions. Every computational resources for packet processing is isolated for each packet and is cleared when execution time limit expires. This prevents malicious programs affecting other packets without virtual machines. Middleware attaches appropriate StreamCode programs considering the user requirements. A sample application, contents-sensitive multicast on our StreamCode emulator, in which a streaming application on a server attaches appropriate multicast program for each contents, optimizes packet loss in multicast in per-user and per-contents basis.
\end{abstract}

\section{Introduction}

End-to-end argument [1] pointed out that some functions must be placed on end user terminals, whether or not equivalent functions are implemented on network nodes. It became a fundamental design principle of the Internet Protocol (IP), and brought us freedom to improve communication functions, e.g., the sophistication of TCP congestion control mechanism or the introduction of URL, new addressing scheme without modifying routers. The freedom made the Internet application friendly, increased innovation speed of applications and contributed to the success of the Internet.

Strange to say, in the very center of this application-oriented network lies a non-service-oriented mechanism: IP. The structure of IP is so simple that it is difficult to introduce application-oriented functions such as QoS or multicast. For QoS some users require strict guarantee, others require loose guarantee, and still others require no guarantee. Some applications allow partial degradation in multicast, others don't. The diversity may exist even in an application. The simpleness and uniformity of IP is not well suited to handle such a wide range of requirements.

Capsule-type active network technology [2] 3] has the potential to be an ideal solution for such requirements. The granularity to define network behavior is packet, small enough for any applications. The definition is done by end users 
(or programmers of end-user applications), thus it satisfies the philosophy of end-to-end argument: give freedom to end users and make network evolution application driven. We therefore believe that capsule should be the basis of the next generation IP or network layer protocol.

However, traditional capsules are too slow to replace IP. ANTS [4] and PLAN 5 uses virtual machine to achieve security, thus can achieve only several tens of Mbps throughput. SNAP 6] developed a sophisticated language to achieve security as well as high-performance. Loops and backward jumps are prohibited, thus the maximum memory consumption and the execution time of a program can be calculated with the program length before execution. The instructions of the language are simple and we may be able to develop dedicated processors to achieve higher performance. However, there are two difficulties in this approach. The first one is that it uses stack to treat variables. This increases the number of memory access, the bottleneck in today's computers. The second one is that the language design policy cannot be applied if we access to the resources on a node, e.g., states in memories. We may therefore have to give up on using states in SNAP packets, which limits the applications.

One common approach to achieve high performance is to develop dedicated hardware. We therefore decided to design capsule system optimized for hardware execution as much as possible, especially security mechanisms. We propose a processor architecture (instruction set and memory management functions) named StreamCode [9] in which program execution of each packet are separated on the basis of time; the processor terminates the execution if a certain time limit expires. We hope we will create multi-gigabit throughput capsule network with this architecture in the future while keeping security necessary for public networks.

Hardware-oriented approaches already exist in active network areas, e.g., Protocol Booster [7] and ANN [8]. In Protocol Booster programmable packet encapsulation is boosted by using FPGAs, and in ANN packet classification between active and traditional packets is accelerated by a chip named APIC. However, they do not give end users the freedom to define network functions which we want to achieve with active network technologies.

This paper is organized as follows. In section 2 we describe the basic design concept and the structure of our StreamCode based active network. In section 3 we discuss the StreamCode prototype system. Then in section 4 we describe the contents-sensitive multi-QoS multicast, an application that demonstrates the power of per-packet QoS customization. Then in section 5 we conclude the paper, discussing remaining issues.

\section{Architectural Description}

We assume two things for in-packet programs.

- Each packet contains the whole program (not program ID) for the packet. This assures that the program is supplied to the StreamCode processor without any sophisticated fetching mechanism. 
- In-packet programs are written in binary format of the processor instruction set. This enables processors to execute the incoming programs immediately.

Figure 1 is a sample in-packet program, hop-by-hop routing written in mnemonic form StreamCode instructions. The program is assembled into binary form and is included in every packet. When a packet with the program arrives at a node, a StreamCode processor in the node starts execution, assuming that the first byte of the packet is the beginning of the program. The packet looks up a routing table named 'DEFAULT_IPv4', allocates memory as an output buffer, copies itself to the buffer and transfers itself to the next node.

Packet Format. Figure 2 shows the packet format. Except for the layer 2 frame (IP header may be treated as this) and ANEP, there is no header, and no clear distinction between the program and user data (the so-called payload). If a programmer wants to describe data in the packet, it should be protected by not executing the area. For example in figure 1 program, destination address field is reserved by skipping the field at the beginning (SKIP $\rightarrow$ real_start). Payload area is secured by terminating program execution before the execution reaches there.

Target Layer. StreamCode instruction set is designed to describe network layer functions, that is, to deliver a packet on end-to-end basis. It is free to use other layer information, for example upper layer information on intermediate nodes (e.g., particular type of users exist in this direction) or lower layer information (e.g., this link is congested) that is provided at nodes. However, the instruction set itself is designed for network layer functions.

Target Plane. In-packet programs written in StreamCode are designed basically for data plane. Since the current difficulty in extending IP functions lies mainly in data plane, the flexibility of capsule technology should focus there. It may also be used for control/management, but the primary target is data plane.

\subsection{Design Concept for High Performance}

To be practical for data plane packet processing, in-packet programs must be processed at comparable throughput and in a secure manner with today's IP. Hereafter in this section we describe the design concept to achieve high performance. The concept for security is described after various components of the network are introduced, in section 2.4

The basic idea that capsule may achieve enough performance to be the foundation of future networks comes from the observation that the speed of today's logical circuit (e.g., CPUs or network processors) is fast enough to achieve multigigabit processing speed. The bottleneck of the processing is, and will be, the access to memories. We therefore made a design decision that we attach the whole program written in binary form StreamCode instructions to each packet, which minimize the memory access during in-packet program execution.

As a result of the decision, all programs and payloads (the data to be handled by the programs) are supplied to the packet-processing unit from incoming port 
// SC sample code for hop-by-hop routing

start:

SKIP $\rightarrow$ real_start;

dest_address: // dest addr.

DATA $\left\{\begin{array}{lllll}192 & 168 & 10 & 05\end{array}\right\}$;

contents_len: // payload's len. w/o this SC

DATA $\left\{\begin{array}{lll}0 & 00\end{array}\right\}$;

real_start:

// IPv4 table lookup; $r(2)$ : output IF id

FUNC TABLE,

table_id=DEFAULT_IPv4,

spc_id $=$ sr (SR_INPUT_SPACE_ID),

addr $=$ dest_address, len $=4 \Rightarrow r(2)$;

SKIP LE, $r(2), 0 \rightarrow$ err;

do_copy:

// copies whole packet: input $\rightarrow$ output

// calculate packet length

MOV4 $i$ (contents_len) $\Rightarrow r(3)$;

ADD $r(3)$, contents $\Rightarrow r(4)$;

FUNC ALLOC, // allocates output buffer

use $=$ OUTPUT, dst_if $=r(2)$,

size $=r(4) \Rightarrow r(5)$;

// actual payload length is here.

SKIP LE, $r(5), 0 \rightarrow$ err;

FUNC COPY,

source_space $=\mathbf{s r}\left(\mathrm{SR}_{-} I N P U T \_S P A C E \_I D\right)$,

source_addr $=0$, length $=r(4)$,

dest_space $=r(5)$,

dest_address $=0 \Rightarrow r(6)$;

do_output: // declaration of packet transfer

// waits until the func copy ends

WAIT $r(6)$;

FUNC OUT_SINGLE,

src_space $=r(5), \quad s r c$ addr $=0$,

src_len $=r(4)$, dst_if $=r(2) \Rightarrow r(7)$;

FIN $r(7)$;

err: // no error handling now.

FIN;

contents:

// here comes actual user data.

Fig. 1. Example StreamCode Program: Hoop-by-hop Routing. 


\begin{tabular}{|c|c|c|}
\hline $\begin{array}{c}\text { L2 } \\
\text { frame }\end{array}$ & ANEP & $\begin{array}{c}\text { Binary format StreamCode } \\
\text { program + user data }\end{array}$ \\
\hline
\end{tabular}

Fig. 2. Packet Format.

and goes out to outgoing port. Since the programs are written in hardwaredecodable instruction set, they can be executed immediately. In an ideal situation where every instruction is executed in a clock and there is no loop or backward jump in the program, the packet goes through the execution unit in a pipeline. The pipeline works at the speed of the network, the same as the required one.

In reality it is impossible to prevent pipeline stall completely. Routing table lookup causes memory access, and some instructions such as the calculation of checksum are almost impossible to complete in a clock. However, thanks to CAM technology it becomes possible to look up a routing table in a fixed clock [10], and using hardware-based parallel processing and sophisticated compiler technology we believe we can limit the effect of the stall.

This approach has two drawbacks. The first one is the fact that lower layer MTU restricts the size of an in-packet program, since it must be in a packet. This makes it impossible to describe complicated algorithms as in-packet programs. The second one is that the same program may be transferred repeatedly in the network, thus wasting the link bandwidth. The sample program in Figure 11 consumes 106 bytes after assembly. If we use cache technology like ANTS [4] or ANN [8], we can relax these problems. However, we believe that if end-user applications can freely attach codes to packets, the number of programs will increase and may reach millions, which makes caching technology ineffective in core routers. Ethernet frame size can easily be expanded to $12 \mathrm{~K}$ octet [1] and the link bandwidth is rapidly increasing thanks to xDSL, WDM and other technologies, which will relax this problem. Complicated algorithms can be realized by putting sophisticated algorithms as node resident programs in EEs (this will be discussed in section 2.2). We therefore decided to accept these drawbacks.

\subsection{Functional Architecture}

Shown in Figure 3 is the architecture of the node designed to handle StreamCode programs in packets. The concept discussed above gives the basic structure for data-path in-packet program processing. Besides the processing environment, environment for control and management plane are necessary in nodes. We therefore decided to put two environments for program execution in a node. One is StreamCode Processing Environment (SC-PE) where in-packet programs are executed. This is for data path functions. The other is Execution Environment (EE) where node-resident programs such as routing daemons are executed. This environment is for control and management functions.

The architecture assumes that a high-end switch is, and will be, composed of a main controller that manages the whole node, intelligent IF cards and a fast 


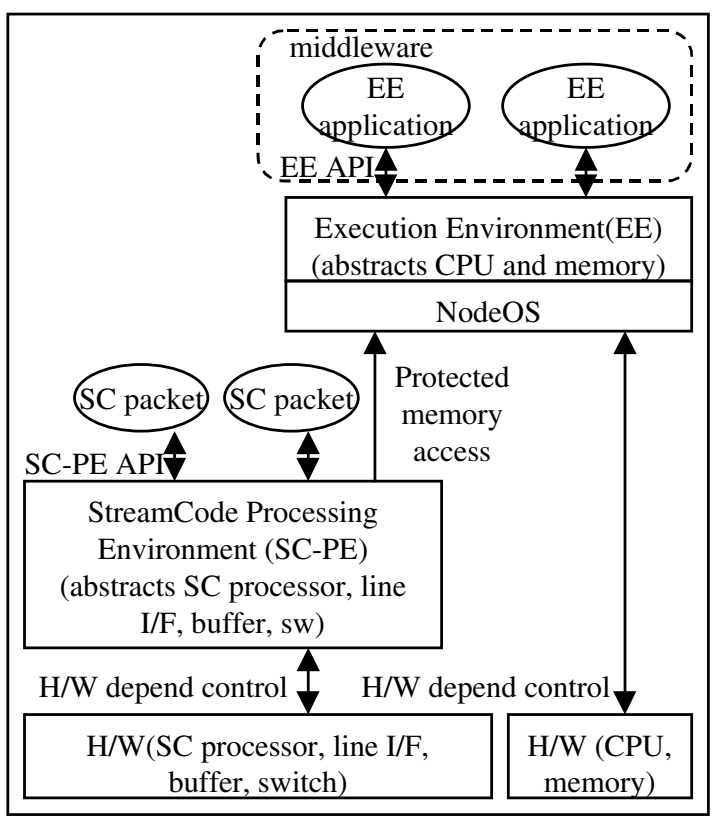

Fig. 3. Functional Architecture of a StreamCode Node.

but dumb switching fabric. A physical image of such a node is shown in Figure 4 Since SC-PE is an abstraction of IF cards and a switch, there may be many $\mathrm{SC}-\mathrm{PEs}$ in a node. EE is an abstraction of the main controller and there is only one EE in a node.

SC-PE. SC-PE is the characteristic environment of this architecture in which incoming in-packet StreamCode programs are executed. Figure 5] shows the internal architecture of SC-PE.

When a packet arrives at a node, it goes into the input buffer through L1/L2 input interface. Then execution unit starts the program execution, using registers, functional units and if necessary, interacting with various memories through Memory Management Unit (MMU). The contents of the input buffer may be copied to output buffer byte by byte, or in a block using a burst-copy function unit. When the output buffer is filled correctly, FUNC OUT_SINGLE or OUT_MULTI instruction (explained in the next subsection) in the program asks the block to move to input queue of the switching module, goes through the switch, and is sent finally to the next node through L1/L2 output interface.

There are two types of memories in SC-PE. One is a temporary memory. This one is initialized on packet arrival and on quit, and can be used solely for the packet. Output buffer is allocated in this type of memory. The other is a permanent memory. Information commonly used among packets, e.g., routing 


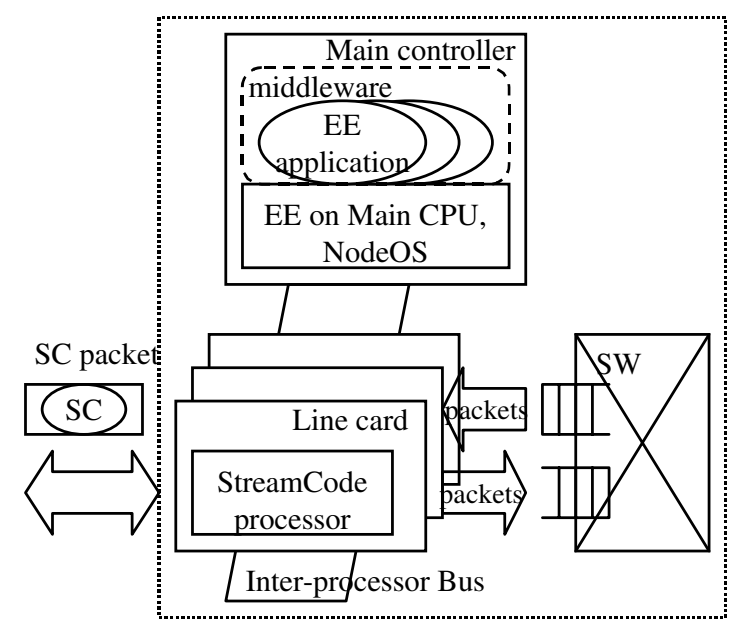

Fig. 4. Physical Image of a StreamCode Node.

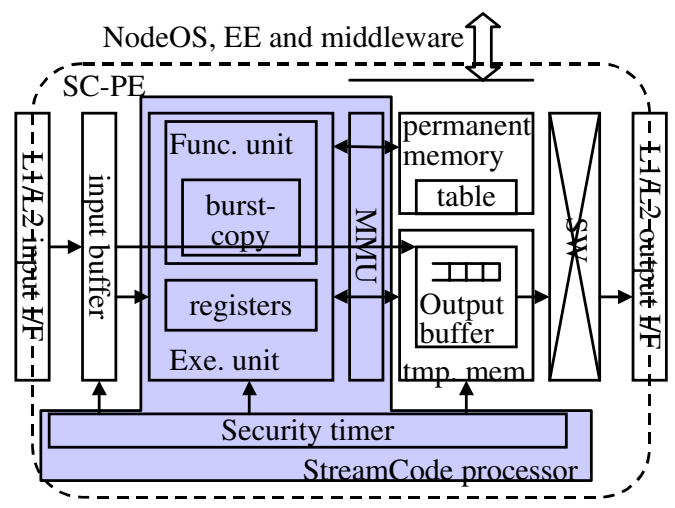

Fig. 5. Internal Architecture of SC-PE.

table information or user request information for contents-sensitive multicast (see section 4) is stored in this area.

There is a security timer that watches input buffer, execution unit and temporary memory. Section 2.4 discusses this.

EE and nodeOS. It is impossible to execute long-lived programs or complicated programs, e.g., routing daemons in SC-PE because execution time for an inpacket program is limited for security reasons (see section 2.4) and the size of the program is limited because of the restriction of MTU.

Most programs for control and management have such characteristics, and EE on nodeOS provides the environment for such programs. These EE and nodeOS 
are equivalent to the one defined in DARPA's active network working group [12]. Using EE programs as the control channel, we can sophisticate algorithms for data path. For example if we need a filtering algorithm that considers congestion level in the network, it can be achieved by running a daemon on EE that collects congestion information and by showing the result to in-packet programs in simple table format.

Interaction between SC-PE and EE. These two environments interact through protected memory access from the SC-PE to the nodeOS. A portion of the main memory of a node is shared memory, and can be looked up from SCPEs as the permanent, read only memory. It may be convenient to allow write access, but since shared memory is not very fast and the access delay fluctuates, which is not desirable for SC-PE, we decided to abandoned it.

There is no particular API to send data from in-packet StreamCode program to nodeOS or programs on EE. If such operation becomes necessary, StreamCode program can do it by making and tossing a small packet to the nodeOS or daemons on the nodeOS as usual packet transfer.

\subsection{StreamCode Instruction Set}

The program for SC-PE is written in StreamCode, a hardware-decodable instruction set. It is composed of basic instructions shown in table 1 and network specific library functions shown in table 2

Table 1. Basic Instructions.

\begin{tabular}{|l|l|}
\hline SC Command & Operation \\
\hline \hline MOV1, MOV2, MOV4 & Load and store primitives \\
\hline $\begin{array}{l}\text { ADD, SUB, } \\
\text { MUL, DIV, MOD }\end{array}$ & Arithmetic/logical calculation \\
\hline SKIP & Conditional/unconditional jump \\
\hline NOP & No operation \\
\hline FIN & Finish program execution \\
\hline FUNC & Call a library function \\
\hline
\end{tabular}

The basic part is a set of RISC-like instructions whose flexibility in describing various programs and scalability in cost, power consumption and performance has already been proved with various implementations. It provides the ability to perform 64 bit memory addressing as well as the access to 256 registers. This is comparable to the instruction sets of current high performance microprocessors, e.g., the Intel IA-64 processor, and we hope that it is enough for future applications. Table 3 shows available memory address sizes, and register numbers for various instruction sets. Such a large number of registers will also make it possible to use them as temporary buffers and contribute to reduce the memory access. 
Table 2. Library Functions.

\begin{tabular}{|l|l|}
\hline Library function & Operation \\
\hline \hline ALLOC & Allocate a buffer in temporary memory \\
\hline ALLOC_PERM & Allocate a buffer in permanent memory \\
\hline FREE & Freeing a buffer in permanent memory \\
\hline COPY & Copy data between buffer \\
\hline TABLE & Looks up a table \\
\hline OUT_SINGLE & Sends a packet to a specified interface \\
\hline OUT_MULTI & Sends packets to specified interfaces \\
\hline
\end{tabular}

Table 3. Available Memory Address and Registers.

\begin{tabular}{|c|c|c|}
\hline & memory address & registers \\
\hline \hline StreamCode & $64 \mathrm{bit}$ & 256 \\
\hline IA-64 & $64 \mathrm{bit}$ & 256 \\
\hline MIPS64 & $64 \mathrm{bit}$ & 64 \\
\hline MIPS32 & $32 \mathrm{bit}$ & 32 \\
\hline
\end{tabular}

Besides basic instructions, StreamCode includes network specific library functions for complicated, time consuming network-specific operations. These functions, e.g., routing table lookup, buffer allocation, burst copy from input buffer to output buffer, etc. will be implemented as a co-processor and will be executed in parallel. The result is placed into a register, and whether a FUNC instruction has been completed and the register is already available or not can be checked from the instruction decoder in the processor. Every time a new instruction is fetched the decoder checks the related registers and if any of them are not ready, the instruction decoding will be blocked and the pipeline will stall. To prevent such stalls programmers should execute several basic instructions after the result of a FUNC instruction becomes necessary.

The reason to develop a new instruction set is to make the instructions variable in length and shrink the size of in-packet programs, whilst still keeping the hardware decoding easy. The StreamCode instruction set can describe the algorithm for contents-sensitive multicast (see section 4) with 384 bytes (table 4), while the MIPS32 instruction set consumes 4110 bytes. The main reason of the huge difference is that StreamCode has a burst, random length DMA copy command that works efficiently in copying payload from input buffer to output buffer. If we assume that MIPS32 also has the command, it consumes 544 bytes, still $41 \%$ larger than StreamCode. Any instructions except FUNCs can be converted to MIPS's in a clock, which shows the easiness of hardware decoding of the instructions. 
Table 4. Program Size of StreamCode, MIPS32, and MIPS32-DMA.

\begin{tabular}{|c|c|c|c|}
\hline Inst. set & Number of Inst. & Program size & average inst. length \\
\hline \hline StreamCode & 119 & 384 bytes & 25.8 bit \\
\hline MIPS32 & 4110 & 16440 bytes & 32 bit \\
\hline MIPS32-DMA & 136 & 544 bytes & 32 bit \\
\hline
\end{tabular}

\subsection{Security}

Since we use a hardware-decodable instruction set, we cannot rely on sophisticated language design for security like ANTS which uses Java virtual machine. We propose a simple security policy:

\section{Limit the execution time for in-packet programs.}

We isolate all the resources for each packet in SC-PE except permanent memory area. When the execution time limit arrives, all the resources used by the packet are cleared. This prevents malfunctioned or malicious packets affecting others. If an in-packet program malfunctions and the packet does not behave as the sender's intention, it is his problem.

The execution time allowed for a packet is the same as the time necessary to receive it; it is proportional to packet length. When a packet arrives at the input buffer of a node, security timer in SC-PE starts. It measures the time to receive the packet and record it. The execution of the in-packet program starts when a certain amount of bit has been stored in the input buffer. When the time to receive the packet has passed from the start of the execution, security timer clears the registers and completely stops the program execution forcefully, except the burst copy from input buffer to output buffer. Programmers therefore must be careful that all the execution except burst copy completes before the time limit arrives. If a packet changes a state on a node incorrectly, it affects all succeeding packets, and the inconsistency must be solved by the application of end users. This may sound too radical, but we believe that this is a correct software evolution because today's processors are becoming more and more dependent on sophisticated compilers.

This security policy has two drawbacks. The first one is the restriction on StreamCode processor implementation. We must standardize the maximum number of clocks for each instruction execution, including load and FUNC instructions to realize this policy. Without this standard a StreamCode programmer cannot know whether or not his program can finish within the time limit. This restricts the implementation, especially the implementation of FUNC instructions. Prefetching of related memories may be necessary. These are the costs to get the freedom to inject codes into networks. The second one is the fact that most packets must contain some payloads. If an in-packet program contains an instruction that does not complete in a clock, payload must be attached to meet 
the security policy whether or not the payload is necessary. This causes the waste of bandwidth because some packets, for example some kinds of ICMP packets do not require payloads. However, as we stated in the performance-related design concept in section 2.1 we believe that the flexibility will become more important than the waste of bandwidth in the future network, and we decided to accept these drawbacks.

\subsection{Session and State Information}

There is a permanent memory region in SC-PE that is not cleared even when the execution time limit for a packet arrives. As described in section 2.2, this region is used for state handling and for information exchange among data packets. We will provide a few authentication mechanisms, from none to cryptographic one to restrict the access to this region. End-user applications should choose appropriate mechanisms considering their risks.

Access right must be set in MMU to use this region. Since it is a timeconsuming task, a user must send a signaling packet beforehand to do this. The packet allocates the required amount of memory and sets an ID to access it. Succeeding data packets show the ID to SC-PE and access to the allocated region.

\subsection{Middleware}

The time-consuming session management signaling will be done by node-resident programs on the EE, though in the current implementation in section 3 it is done by normal in-packet StreamCode programs.

We believe that there should be various node-resident programs, or middleware on end-user terminals and on intermediate nodes. The functional difference between terminals and intermediate nodes is the difference of the programs. On end-user terminals a typical function is an advanced socket emulation by attaching appropriate StreamCode programs to appropriate packets considering network congestion, failure and user requirements. On intermediate nodes (general/proprietary) routing daemons, user authentication for session management and logging are common functions.

\section{Proof-of-Concept Implementation}

StreamCode is designed to be implemented in hardware, but we made a proofof-concept prototype on FreeBSD 4.2 in software because of the easiness of implementation.

The primary purpose of this prototype is to check whether it is possible to write meaningful applications such as contents-sensitive multicast (see section 4) with reasonable program length or the number of instructions. The prototype is therefore a minimum set to achieve this goal and is composed of SC-PE and its minimum interface for packet exchange, i.e., packet receive and send. 


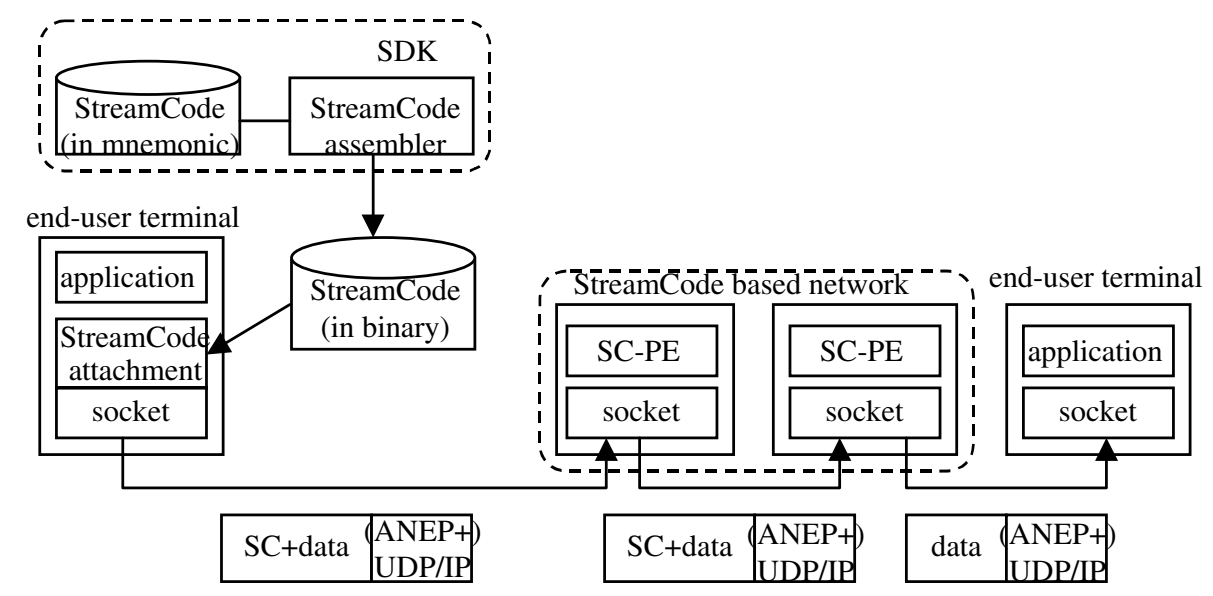

Fig. 6. Overall Architecture of Experimental System.

We call this SC engine. Other components and interfaces such as nodeOS, EE, middleware and the interaction between them will be implemented in the future. The program execution termination mechanism is also omitted. Whether the packet execution can finish within the time limit can be calculated from the standardized maximum instruction execution time, and the data for standardization should come from hardware implementation. We therefore did not implement the execution termination mechanism in the current version.

Figure 6] shows the architecture overview of the prototype.

The development flow of StreamCode applications and StreamCode packet processing are as follows.

1. A programmer writes StreamCode programs in mnemonic considering the user- and application-specific requirements. They are assembled and are stored in binary form.

2. A StreamCode attachment module on a server integrates a stored binary program and a payload into a complete StreamCode packet.

3. Current implementation uses UDP/IP as the layer two of StreamCode packets. ANEP implementation is ongoing. The StreamCode programs and user data become payloads of UDP/IP packets and sent into the network.

$\mathrm{UDP} / \mathrm{IP}$ is used just for datalink layer. Routing must be done by StreamCode program.

4. When a node receives a packet, it executes the in-packet StreamCode program and, if required, transfer it to the next node by changing the destination address in the UDP/IP header.

5. There is a special register in a StreamCode processor that shows whether the node is the edge of a StreamCode network or not. If a StreamCode program wants to deliver normal UDP/IP packet to the destination, this can be done 


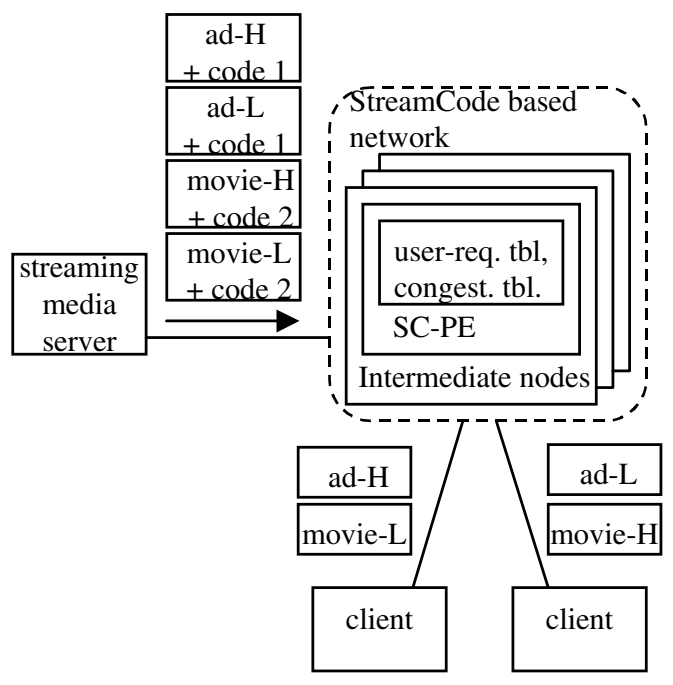

Fig. 7. Overall Architecture of Contents-Sensitive Multi-QoS Multicast.

by not copying program part of the packet when the packet is in the node next to destination.

If we use Intel Pentium III 933MHz processor and Netgear GA620 1000BaseSX NIC as the hardware and execute a hop-by-hop packet forwarding program, the SC-PE processes 6223 packets/sec, whose packet size is 8746 bytes. It corresponds to $829 \mathrm{Mbps}$ throughput.

\section{Contents-Sensitive Multi-QoS Multicast}

Using StreamCode we can customize each packet's processing algorithm and thus customize each packet's QoS by attaching a different program to each packet. Contents-sensitive multicast is an application that shows the potential of this flexibility.

Figure 7 shows the architecture of the system. A streaming media server multicasts movies and advertisements in serial form like today's TV channels. For each video two or three streams of different quality are simulcasted, e.g., low quality movie and high quality movie. Different StreamCode programs are attached to movie packets and advertisement packets.

There are two tables on intermediate StreamCode nodes. One is an application specific table, that is, a table that describes the requirements of users connected to each output port. Some users require high-quality movie because they are willing to pay for that. Some users require low-quality movie because they receive it on PDAs. For a user who does not pay, sponsor of the advertise- 
ment requests the network to deliver the ad in high quality. The other table is a general congestion table of that output port.

When a movie packet arrives at an intermediate StreamCode node, the program checks the quality identifier in the payload and looks up the user requirements table. If the packet contains the same or lower than the quality requested by users of a port, it checks the congestion level and if the level is low enough for the packet to be transferred, it goes to the next node with the program. If the node is at the edge of an active network, the packet transfers itself to the next node (probably the user terminal) if it contains the highest quality video which manages to reach there in the congestion. On transfer for the last hop, current program is designed not to copy the program part of itself, thus the packets that the user terminals receive are traditional UDP/IP packets and can be played back with ordinary streaming media players. Advertisement packets behave in the same manner, though they look up different fields of the requirement table.

The reason why we use simulcast of different quality video is the easiness of implementation. If we use more sophisticated, hierarchical encoding schemes such as WaveVideo [13, we can unify these streams.

We implemented this application on the prototype described in the previous chapter. It worked well with MicroSoft Media Player as the client software.

As mentioned in table 4 it consumes 384 bytes to describe this algorithm. This is not a small value, but we believe that this is acceptable as the cost of user-driven network evolution when MTU is extended to $9 \mathrm{~K}$ bytes or more. We therefore conclude that in-packet programs in StreamCode can describe practical programs under MTU limitation.

\section{Conclusion and Future Plan}

We proposed a capsule type active network architecture and the processor architecture, StreamCode, whose security is achieved by limiting in-packet program execution on time-basis. Using a proof-of-concept implementation, we also proposed a contents-aware multicast whose QoS is controlled on per-packet basis.

There are many issues which have to be solved. One of the most important is implementing SC-PE in hardware, through which to prove the applicability of the security policy as well as to prove the competitiveness in performance with traditional IP routers.

From the QoS control point of view, we plan to investigate the possibility of per-packet QoS customization further. Current method uses only the abstracted congestion level. It may contain various information such as failures or congestion levels of particular geographical areas. Packet handling method can also be improved. If congestion occurs packets are discarded as a whole now, but partial discard of a payload may make sense. We would like to evaluate these possibilities. 


\section{References}

1. H. Saltzer, D. P. Reed, and D. Clark, "End-to-end arguments in system design", ACM Transactions on Computing Systems, vol. 2, no. 4, 1984.

2. D.L. Tennenhouse, J.M. Smith, D. Sincoskie, D.J. Wetherall, and G. Minden, "A Survey of Active Network Research", IEEE Communications, Jan. 1997.

3. K. Psounis, "Active Networks: Applications, Security, Safety, and Architectures", IEEE Communications Survey, Vol2, No.1., 1st quarter 1999.

4. D.J. Wetherall, J. Guttag, and D.L. Tennenhouse, "ANTS: A Toolkit for Building and Dynamically Deploying Network Protocols", in Proceedings IEEE OPENARCH'98, April 1998.

5. M. Hicks, J. Moore, D. Alexander, C. Gunter, and S. Nettles, "PLANet: An Active Internetwork", in Proceedings INFOCOM'99, New York, 1999.

6. J. Moore, M. Hicks, and S. Nettles, "Practical Programmable Packets", in Proceedings INFOCOM 2001, April 2001.

7. D.C. Feldmeier, A.J. McAuley, J.M. Smith, D. Bakin, W.S. Marcus, and T. Raleigh, "Protocol Boosters", IEEE JSAC, vol 16, no. 3, pp. 437-444, April 1998.

8. D. Decasper, G. Parulkar, S. Choi, J. DeHart, T. Wolf, and B. Plattner, "A Scalable, High Performance Active Network Node", in IEEE Network, January/February 1999.

9. K. Hino, T. Egawa, and Y. Kiriha, "Open Programmable Layer-3 Networking", in Proceedings SmartNet2000, September 2000.

10. M. Kobayashi, T. Murase, and A. Kuriyama, "A Longest Prefix Match Search Engine for Multi-Gigabit IP Processing", in Proceedings IEEE ICC 2000, June 2000.

11. P. Dykstra, "Gigabit Ethernet Jumbo Frames And why you should care", December 1999. http://63.196.71.246/ phil/jumbo.html

12. K.L. Calvert (ed.), "Architectural Framework for Active Networks Version 1.0", DARPA active network working group, July 27, 1999.

13. R. Keller, S. Choi, M. Dasen, D. Decasper, D. Fankhauser, and B. Plattner, "An Active Router Architecture for Multicast Video Distribution", in Proceedings Infocom 2000, March 2000. 